\title{
The association between osteopontin and survival in non-small-cell lung cancer patients: a meta-analysis of I 3 cohorts
}

This article was published in the following Dove Press journal:

OncoTargets and Therapy

26 November 2015

Number of times this article has been viewed

\author{
Ying Wangl,* \\ Jin Yangl,* \\ Hui Liu' \\ Ji-Rui Bi \\ Ying Liu $^{2}$ \\ Yan-Yan Chen ${ }^{2}$ \\ Ji-Yu Cao ${ }^{2}$ \\ You-Jin Lu' \\ 'Department of Respiratory \\ Medicine, the Second Affiliated \\ Hospital of Anhui Medical University, \\ ${ }^{2}$ Department of Occupational Health \\ and Environmental Health, School \\ of Public Health, Anhui Medical \\ University, Hefei, People's Republic \\ of China \\ *These authors contributed equally \\ to this work
}

\begin{abstract}
In the last decade, osteopontin (OPN) was identified as one of the important proteins that promote the metastasis of tumor. However, the association between OPN overexpression and clinical outcome of non-small-cell lung cancer (NSCLC) was unclear. The purpose of this study is to investigate the role of OPN in NSCLC patients. A total of 13 studies are included to explore the relationship between the OPN elevation and the overall survival (OS) and disease-free survival (DFS) in NSCLC patients. We searched for related articles in PubMed, Web of Science, Google Scholar, and Cochrane Library databases, which were published before January 31, 2015. Hazard ratio (HR), odds ratio (OR), and 95\% confidence interval (CI) in the high OPN expression group compared with the low OPN expression group were calculated and analyzed. Primary results were summarized by using a fixed-effects model or a random-effects model. The stratified analyses in subgroups were also performed. Thirteen cohort studies, which involved 1,630 patients, were included. Subgroup analyses were performed by area and test method of OPN. We found that OPN was significantly associated with poor OS (HR $=2.20,95 \%$ CI 1.71-2.83, $P<0.001)$ and DFS (HR $=2.11,95 \%$ CI $1.62-2.74$, $P<0.001)$ in NSCLC patients. OPN overexpression tended to be associated with the presence of advanced tumor TNM stage (III and IV) (OR $=2.57,95 \%$ CI $1.61-4.11, P<0.001)$. The Egger's test suggested that there was no publication bias in OS studies $(P=0.062)$ and DFS studies $(P=0.740)$. These data indicate that OPN seems to have a significant predictive potential in estimating survival in NSCLC.
\end{abstract}

Keywords: metastasis, overall survival, disease-free survival, tumor stage

\section{Introduction}

Lung cancer is one of the most frequently diagnosed solid tumors in the world as well as the most common causes of cancer mortality worldwide, with an overall 5-year survival rate of $15 \%$. ${ }^{1}$ Non-small-cell lung cancer (NSCLC) accounts for $\sim 80 \%-85 \%$ of all lung cancers. ${ }^{2}$ In spite of early detection and obvious improvements in surgical techniques, the postoperative recurrence rate is higher in lung cancer compared to other types of cancer. ${ }^{3}$ As the current TNM staging system for NSCLC does not provide satisfactory prognostication, it is essential to identify the novel biomarkers that will contribute to recognize a high risk of metastasis and recurrence of patients. Moreover, novel therapies are certainly warranted in NSCLC, and as targeted treatment is becoming important gradually, identifying molecular markers as potential therapeutic targets is urgent.

Osteopontin (OPN) is a $32-\mathrm{kDa}$ multifunctional protein with features of both a matrix protein and a cytokine, which is involved in tissue remodeling, malignant transformation, and immune-mediated response. Furthermore, OPN was found to be frequently overexpressed in many solid tumors, such as breast, hepatocellular,
Correspondence: You-jin Lu Department of Respiratory Medicine, the Second Affiliated Hospital of Anhui Medical University, No 678, Fu Rong Road, Hefei 23060I, Anhui Province, People's Republic of China Fax +86 55I 63869593

Email luyougolden@hotmail.com 
gastric, colorectal, and lung carcinomas. ${ }^{4-7}$ Although the correlation between OPN and tumor has been discussed for several years, the existing data have not been analyzed thoroughly till now. It has been demonstrated that OPN is associated with cancer development, progression, and metastasis in different malignancies, including NSCLC. ${ }^{5-10}$ An overexpression of OPN has been found to occur not only in pathologic conditions, such as ischemia or inflammation, but also in many types of malignancies. Recent clinical and experimental studies suggested that overexpression of OPN in NSCLC patients may correlate with disease stage ${ }^{9-11}$ and survival. ${ }^{12,13}$ However, the clinical implications of OPN in NSCLC patients have not been fully investigated.

Therefore, it is necessary to conduct a meta-analysis to systematically understand the relationship between OPN and survival in NSCLC. The aim of this study is to evaluate the significance of serum- and elevated tissue-based OPN levels for overall survival (OS) and disease-free survival (DFS) in patients with NSCLC.

\section{Materials and methods Publication search}

Relevant literatures were identified by searching the PubMed, Web of Science, Google Scholar, and Cochrane Library databases before January 31,2015 . The search strategy was based on the following keywords: osteopontin or OPN, lung cancer or pulmonary carcinoma, and survival. Articles written in English were all eligible for inclusion. In addition, in order to omit any other relevant studies, the references to all identified publications were also searched, and investigators were contacted to supply additional data when key information relevant to the meta-analysis was missing.

\section{Inclusion and exclusion criteria}

Studies were included if they met the following criteria: 1) measured OPN expression in the NSCLC patients with enzyme-linked immunosorbent assay (ELISA), polymerase chain reaction (PCR), or immunohistochemistry (IHC); 2) provided information on survival; and 3) provided the hazard ratio (HR) and $95 \%$ confidence interval (CI) or raw data. The excluded criteria were as follows: review articles, laboratory studies, animal studies, and case reports.

\section{Quality assessment}

Quality assessment was carried out in the available studies using the Newcastle-Ottawa quality assessment scale for cohort studies by two researchers independently (Table 1). ${ }^{14}$ The scores range from 0 to 9 . When publications had $\geq 6$ scores, they were graded as the high-quality ones.
Table I Newcastle-Ottawa quality assessment scale for cohort studies

\section{Selection}

1) Representativeness of the exposed cohort

a) Truly representative of the average NSCLC patients (describe) in the community $(*)$

b) Somewhat representative of the average NSCLC patients in the community $(*)$

c) Selected group of users, eg, nurses, volunteers

d) No description of the derivation of the cohort

2) Selection of the nonexposed cohort

a) Drawn from the same community as the exposed cohort (*)

b) Drawn from a different source

c) No description of the derivation of the nonexposed cohort

3) Ascertainment of exposure (proof of NSCLC and osteopontin measurement)

a) Secure record (eg, surgical records) (*)

b) Structured interview $(*)$

c) Written self-report

d) No description

4) Demonstration that outcome of interest was not present at start of study

a) Yes (*)

b) No

\section{Comparability}

1) Comparability of cohorts on the basis of the design or analysis

a) Study controls for recurrence or metastasis (select the most important factor) $(*)$

b) Study controls for any additional factor (age, stage, grade, cirrhosis, etc) $(*)$

\section{Outcome}

1) Assessment of outcome

a) Independent blind assessment (*)

b) Record linkage $(*)$

c) Self-report

d) No description

2) Was follow-up long enough for outcomes to occur

a) Yes (2 years) (*)

b) No

3) Adequacy of follow-up of cohorts

a) Complete follow-up - all subjects accounted for $(*)$

b) Subjects lost to follow-up unlikely to introduce bias - small number lost $>25 \%$ (select an adequate\%) follow-up, or description provided of those lost) $(*)$

c) Follow-up rate $<75 \%$ (select an adequate\%) and no description of those lost

d) No statement

Notes: A study can be awarded a maximum of one star for each numbered item within the selection and outcome categories. A maximum of two stars can be given for comparability.

Abbreviation: NSCLC, non-small-cell lung cancer.

\section{Definitions and data extraction}

OS was defined as the time interval between the medical treatment (including lung resection, chemotherapy, radiotherapy, etc) and death or the last observation. DFS was measured from the date of treatment to the recurrence or the last assessment. Data were carefully extracted from all eligible publications independently by two authors 
(YW and JY) based on the inclusion and exclusion criteria. Disputes were settled through friendly consultations. But if necessary, the third author (HL) could be consulted to help settle disputes. The following variables in each study were extracted: 1) primary author's name and year of publication; 2) characteristics of the study population (sex and number); 3) the measurement method of OPN; and 4) tumor stage, follow-up time, and the outcome of patients.

\section{Statistical analysis}

All statistical analyses were carried out with Stata/SE, Version 10 (StataCorp LP, College Station, TX, USA). For the assessment of the association between OPN overexpression and survival in patients with NSCLC, the HR and 95\% CI were extracted from studies or calculated with the raw data if provided. For the assessment of the association between OPN overexpression and tumor stage in patients with NSCLC, the odds ratio (OR) and 95\% CI were extracted from studies. In the subgroup analyses, we explored the effects of different testing methods and crowd from different areas on the strength of the association between OPN and survival results. Statistical heterogeneity was measured using the chi-square test and $I^{2}$ test. If the $I^{2}$ value is $<50 \%$ or the $P$-value is $>0.10$, significant heterogeneity will not be considered. ${ }^{15}$ A random-effects model was used for pooled analyses when between-study heterogeneity was substantial; otherwise, a fixed-effects model was used. We conducted the Egger's test to evaluate the publication bias of eligible studies, and publication bias was considered when a $P$-value was $<0.05{ }^{16}$

\section{Results}

\section{Selection and characteristics of studies}

By the initial literature search, 163 relevant articles were identified regarding the association of OPN and NSCLC. After the careful screening process, 120 studies were excluded as they were review articles, experimental research, and studies irrelevant to the current analysis. Forty-three relevant studies were selected for detailed evaluation. Of the remaining 43 studies, 30 publications did not meet the inclusion criteria and were excluded. Finally, a total of 13 studies $^{7,10,12,13,17-25}$ with 1,630 patients were included in our meta-analysis (Figure 1). Four studies were performed in People's Republic of China, ${ }^{17,20,22,25}$ two in Japan, ${ }^{12,13}$ two in America, ${ }^{10,21}$ one in Norway, ${ }^{19}$ one in England, ${ }^{18}$ one in Italy, ${ }^{7}$ one in Germany, ${ }^{24}$ and one in Korea. ${ }^{23}$ The sample size of each study varied from 25 to 244 . Seven studies used IHC, five used ELISA, and one used PCR. The quality of study was assessed by Newcastle-Ottawa quality assessment scale, whose scores ranged from 5 to 7 (median $=6$ ). The characteristic of each article included in this meta-analysis is revealed in Table 2 .

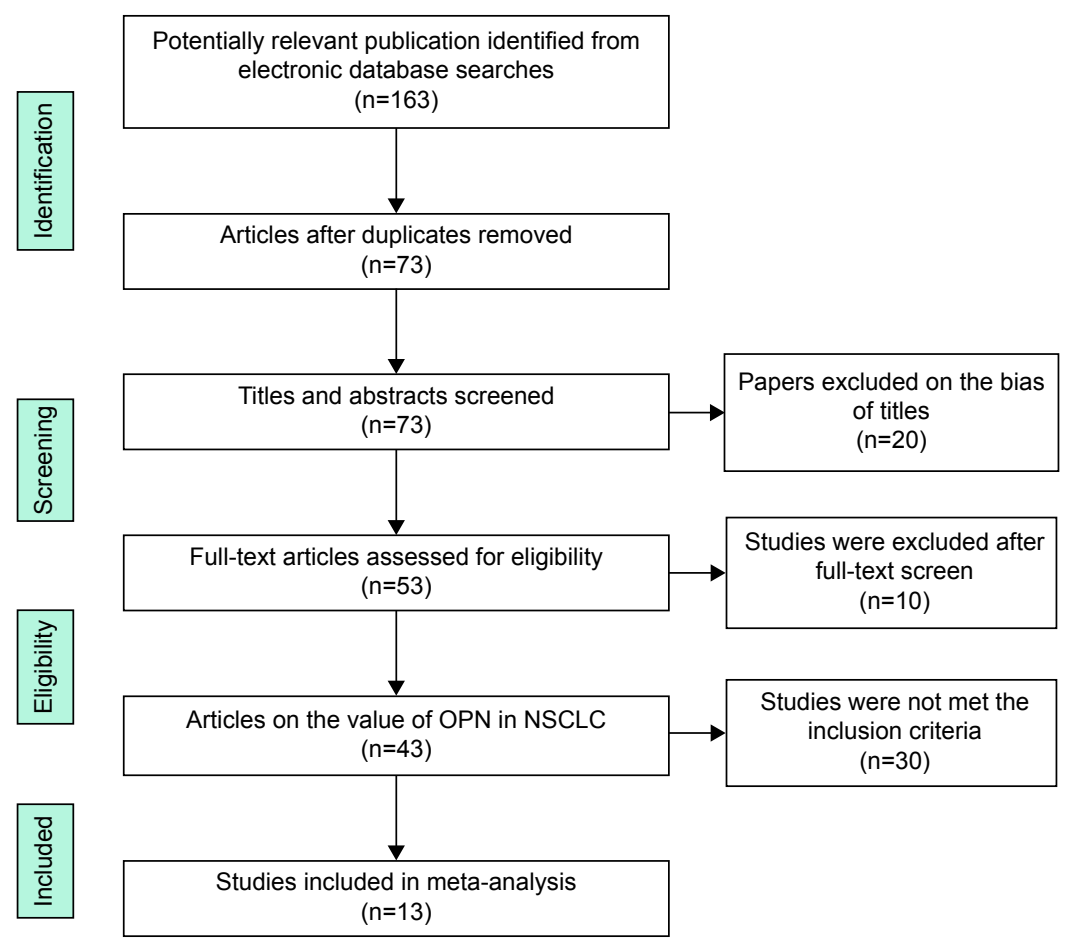

Figure I Flow diagram of the study selection process.

Abbreviations: OPN, osteopontin; NSCLC, non-small-cell lung cancer. 


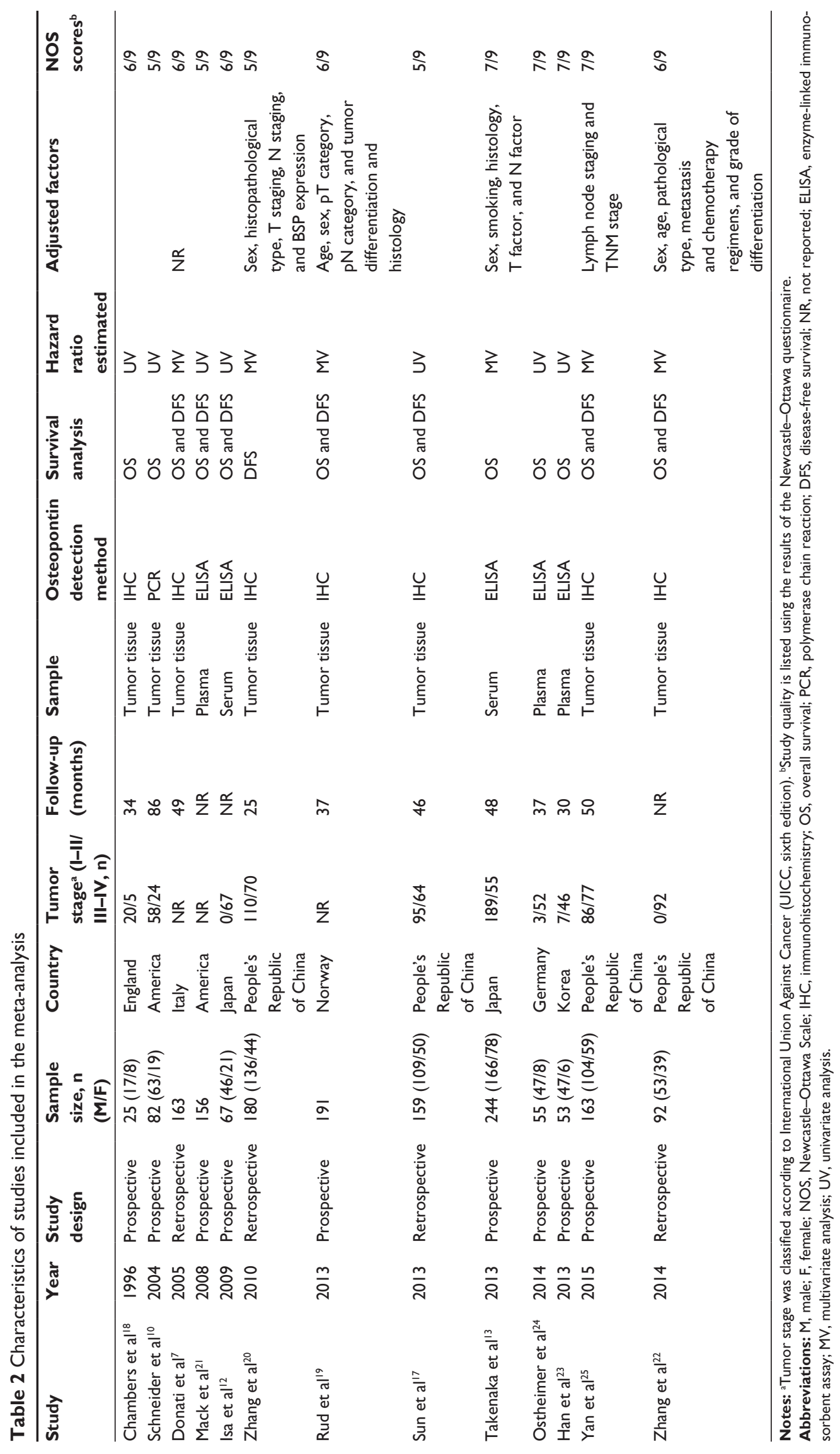




\section{OPN expression and OS in NSCLC}

A total of 12 studies, which included 1,450 patients, reported data on OPN expression and OS in NSCLC. The sample size of each study varied from 25 to 244 . OPN levels were detected by IHC in six articles - five by ELISA and one by PCR. OPN overexpression seemed to be correlated with poor OS in all studies (Figure 2). Combined data from all the 12 studies showed that increased OPN levels were associated with OS with a pooled HR estimate of 2.20 (95\% CI $1.71-2.83, P<0.001)$, with significant heterogeneity in the data $\left(I^{2}=69.6 \%, P<0.001\right)$.

Subgroup analyses were performed by area and test method of OPN. For area, the analysis was stratified into two subgroups: non-Asia and Asia. A significantly increased risk was found in Asia (HR $=2.68,95 \%$ CI 1.63-4.43, $P<0.001)$ and also in non-Asia (HR $=1.66,95 \%$ CI $1.43-1.93$, $P<0.001)$. For the test method of OPN, the analysis was stratified into two subgroups: ELISA and IHC. A significantly increased risk was found by ELISA (HR $=1.80,95 \%$ CI $1.43-2.27, P<0.001)$ and also by IHC (HR $=2.63,95 \% \mathrm{CI}$ $1.65-4.22, P<0.001$ ) (Table 3 ). The result of analysis whose subgroup stratified into tumor tissue was $\mathrm{HR}=2.50,95 \%$ CI 1.68-3.73, $P<0.001$; into plasma was $\mathrm{HR}=1.71,95 \%$ CI 1.28-2.28, $P<0.001$; and into serum was $\mathrm{HR}=1.98$, $95 \%$ CI $1.35-2.92, P=0.001$.

\section{OPN expression and DFS in NSCLC}

Eight studies reported data on OPN expression and DFS in NSCLC. A high OPN expression was significantly correlated with poor DFS in all studies (Figure 3). Pooled data from all the eight studies showed that increased OPN levels were associated with DFS with a pooled HR estimate of 2.11 (95\% CI 1.62-2.74, $P<0.001$ ), with significant heterogeneity in the data $\left(I^{2}=50.3 \%, P=0.050\right)$.

The result of analysis whose subgroup stratified into Asia was $\mathrm{HR}=2.40,95 \%$ CI $1.86-3.09, P<0.001$ and into nonAsia was HR $=1.74,95 \%$ CI 1.10-2.76, $P=0.017$. For test method of OPN (Table 3), likewise, a significantly increased risk was found by ELISA (HR $=1.79,95 \%$ CI 1.11-2.86, $P=0.016)$ and also by IHC (HR $=2.36,95 \%$ CI $1.89-2.96$, $P<0.001)$. For sample type, a significantly increased risk was found in tumor tissue (HR $=2.36,95 \%$ CI $1.89-2.96$, $P<0.001)$.

\section{OPN expression and tumor stage in NSCLC}

Three studies reported data on OPN expression and tumor TNM stage in NSCLC. ${ }^{17,24,25}$ High OPN expression seemed to be associated with high tumor stage (III and IV) in two studies and low tumor stage in one study. Combined data from all the three studies showed a trend for a correlation between

\begin{tabular}{|c|c|c|c|}
\hline Study ID & & HR (95\% CI) & $\%$ weight \\
\hline Chambers et al ${ }^{18}$ & $\rightarrow$ & $1.50(1.22,1.84)$ & 11.53 \\
\hline Schneider et al ${ }^{10}$ & $\rightarrow$ & $1.93(1.24,3.01)$ & 9.01 \\
\hline Donati et al ${ }^{7}$ & & $1.48(0.79,2.77)$ & 7.05 \\
\hline Mack et $\mathrm{al}^{21}$ & $\rightarrow$ & $1.67(1.19,2.33)$ & 10.22 \\
\hline Isa et al ${ }^{12}$ & & $1.75(1.03,3.03)$ & 7.95 \\
\hline Rud et al ${ }^{19}$ & & $2.70(1.20,6.30)$ & 5.32 \\
\hline Rud et al ${ }^{19}$ & & $2.80(1.20,6.50)$ & 5.20 \\
\hline Sun et al ${ }^{17}$ & & $2.35(1.54,3.58)$ & 9.25 \\
\hline Takenaka et al ${ }^{13}$ & & $2.26(1.30,3.94)$ & 7.79 \\
\hline Ostheimer et al ${ }^{24}$ & & $2.20(1.10,4.80)$ & 6.05 \\
\hline Han et $a^{23}$ & & $1.43(0.62,3.29)$ & 5.28 \\
\hline Yan et al ${ }^{25}$ & & $2.41(1.51,3.83)$ & 8.78 \\
\hline Zhang et $\mathrm{al}^{22}$ & & $12.01(6.11,23.59)$ & 6.59 \\
\hline Overall $\left(I^{2}=69.6 \%, P=0.001\right)$ & & $2.20(1.71,2.83)$ & 100 \\
\hline
\end{tabular}

Figure 2 Meta-analysis of the association between OPN overexpression and OS of NSCLC

Notes: The summary HR and $95 \% \mathrm{Cls}$ were also shown (according to the random-effect estimations). Weights are from random-effects analysis. Abbreviations: OPN, osteopontin; OS, overall survival; NSCLC, non-small-cell lung cancer; $\mathrm{HR}$, hazard ratio; Cl, confidence interval. 
Table 3 Subgroup analysis of the OPN levels in NSCLC patients

\begin{tabular}{|c|c|c|c|c|c|c|c|}
\hline Outcome & Variable & $\mathbf{N}$ & Model & HR (95\% CI) & $P$-value & $I^{2}(\%)$ & $\boldsymbol{P}_{\text {heterogeneity }}$ \\
\hline \multirow[t]{12}{*}{ OS } & All & 12 & Random & $2.20(1.71,2.83)$ & $<0.001$ & 69.6 & $<0.001$ \\
\hline & Area & & & & & & \\
\hline & Non-Asia & 6 & Fixed & $1.66(1.43,1.93)$ & $<0.001$ & 0.0 & 0.562 \\
\hline & Asia & 6 & Random & $2.68(1.63,4.43)$ & $<0.001$ & 79.4 & $<0.001$ \\
\hline & Method & & & & & & \\
\hline & ELISA & 5 & Fixed & $1.80(1.43,2.27)$ & $<0.001$ & 0.0 & 0.840 \\
\hline & $\mathrm{IHC}$ & 6 & Random & $2.63(1.65,4.22)$ & $<0.001$ & 84.1 & $<0.001$ \\
\hline & PCR & I & & $1.93(1.24,3.01)$ & & & \\
\hline & Sample type & & & & & & \\
\hline & Tumor tissue & 7 & Random & $2.50(1.68,3.73)$ & $<0.001$ & 81.4 & $<0.001$ \\
\hline & Plasma & 3 & Fixed & I.7I $(1.28,2.28)$ & $<0.001$ & 0.0 & 0.724 \\
\hline & Serum & 2 & Fixed & $1.98(1.35,2.92)$ & 0.001 & 0.0 & 0.521 \\
\hline \multirow[t]{11}{*}{ DFS } & All & 8 & Random & $2.11(1.62,2.74)$ & $<0.001$ & 50.3 & 0.050 \\
\hline & Area & & & & & & \\
\hline & Non-Asia & 3 & Fixed & $1.74(1.10,2.76)$ & 0.017 & 44.4 & 0.166 \\
\hline & Asia & 5 & Fixed & $2.40(1.86,3.09)$ & $<0.001$ & 20.3 & 0.285 \\
\hline & Method & & & & & & \\
\hline & ELISA & 2 & Random & $1.79(1.11,2.86)$ & 0.016 & 59.8 & 0.115 \\
\hline & $\mathrm{IHC}$ & 6 & Fixed & $2.36(1.89,2.96)$ & $<0.001$ & 37.2 & 0.158 \\
\hline & Sample type & & & & & & \\
\hline & Tumor tissue & 6 & Fixed & $2.36(1.89,2.96)$ & $<0.001$ & 37.2 & 0.158 \\
\hline & Plasma & I & & $1.46(1.05,2.00)$ & & & \\
\hline & Serum & I & & $2.38(1.43,4.00)$ & & & \\
\hline
\end{tabular}

Abbreviations: OPN, osteopontin; NSCLC, non-small-cell lung cancer; $\mathrm{HR}$, hazard ratio; $\mathrm{Cl}$, confidence interval; OS, overall survival; ELISA, enzyme-linked immunosorbent assay; IHC, immunohistochemistry; PCR, polymerase chain reaction; DFS, disease-free survival.

high OPN expression and high tumor TNM stage (III and IV) (OR $=2.57,95 \%$ CI 1.61-4.11, $P<0.001)$ (Figure 4) without any heterogeneity $\left(I^{2}=0 \%, P=0.417\right)$. Regrettably, only two studies provided relevant data on the correlation of OPN with tumor grade. ${ }^{22,24}$ The pooled analysis could not be performed.

\section{Publication bias}

Publication bias was mainly used to evaluate the reliability of meta-analysis results, especially those that showed statistical significance. ${ }^{7}$ The Egger's test (statistical significance was set at $P<0.05)$ indicated that publication bias was absent

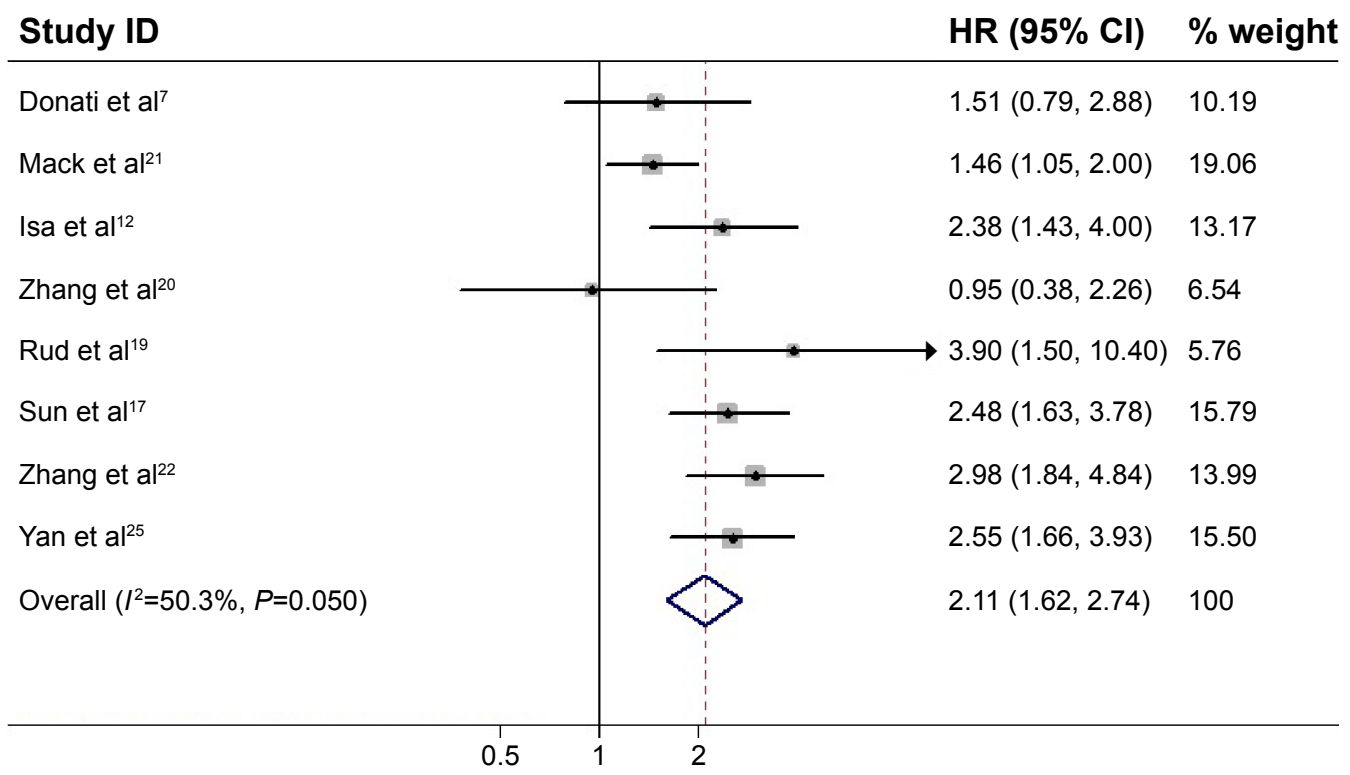

Figure 3 Meta-analysis of the association between OPN overexpression and DFS of NSCLC.

Notes: The summary HR and 95\% Cls were also shown (according to the random-effect estimations). Weights are from random-effects analysis. Abbreviations: OPN, osteopontin; NSCLC, non-small-cell lung cancer; DFS, disease-free survival; HR, hazard ratio; Cl, confidence interval. 


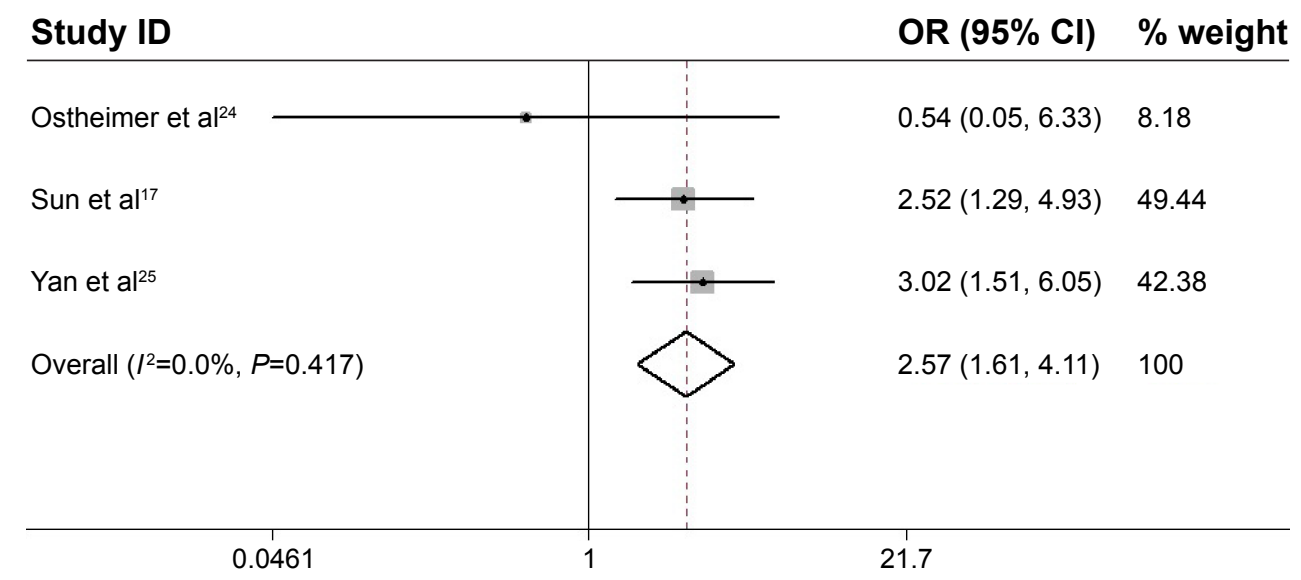

Figure 4 Meta-analysis of the association between OPN overexpression and tumor TNM stage of NSCLC.

Note: The summary OR and $95 \% \mathrm{Cls}$ were also shown (according to the fixed-effect estimations).

Abbreviations: OPN, osteopontin; NSCLC, non-small-cell lung cancer; OR, odds ratio; CI, confidence interval.

in studies on OPN associated with OS $(P=0.062)$ and DFS $(P=0.740)$.

\section{Sensitivity analysis}

To assess the stability to the results of this meta-analysis, we performed a sensitivity analysis by excluding each study sequentially. Statistically similar results were obtained after excluding each study sequentially, which indicates the stability to this meta-analysis.

\section{Discussion}

Despite significant advances in supervising and clinical treatment strategies, the overall prognosis of NSCLC remains dismal. ${ }^{26-30}$ It is important to classify the patients with a high possibility of tumor recurrence and metastasis in order to initiate intervention in time. The existing TNM stage cannot predict the outcome of all NSCLC patients accurately. Therefore, identifying effective biomarkers involved in tumor spreading and survival is an essential step to improve the prognosis of this disease. ${ }^{9,31-33}$

In this article, we first evaluated the association between high OPN expression and survival in NSCLC patients. The pooled results demonstrated that high OPN expression significantly predicted poor OS and DFS for NSCLC $(P<0.001$ and $P<0.001$, respectively). After subgroup analyses using the detection methods, OPN elevation was significantly associated with OS and DFS in patients who were tested by ELISA ( $P<0.001$ and $P<0.001$, respectively) and by IHC $(P<0.001$ and $P<0.001$, respectively). When the subgroup was stratified separately according to area, OPN overexpression was significantly associated with OS of patients in nonAsia $(P<0.001)$ and also in Asia $(P<0.001$ and $P<0.001$, respectively).
The reasons for the diversities of OS and DFS among NSCLC patients with high and low OPN expressions were still unclear. Approximately two-thirds of recurrences for early stage cancer after surgery occurred on distant sites. Therefore, the elimination of micrometastases should be given a high priority as soon as possible. OPN played an essential role in tumorigenesis, progression, and metastasis in NSCLC, and patients in TNM stage II or III younger than 70 years received adjuvant chemotherapy after surgery, in which OPN-negative patients showed a good outcome as their 3 -year relapse-free survival was similar to stage I patients younger than 70 years. ${ }^{19}$ Whereas the OPN-positive patients showed an obviously poor outcome, tumor OPN expression was an unfavorable prognostic factor not only for cases of advanced NSCLC but also for patients with stage I of the disease. ${ }^{13}$ They also pathologically evaluated the association between OPN levels and tumor invasiveness. They found that a higher OPN level was particularly associated with higher incidences of vascular invasions, suggesting that patients with NSCLC with high OPN levels had a more aggressive tumor phenotype. Ostheimer et $\mathrm{al}^{134}$ reported in a trial of 69 patients with inoperable NSCLC that OPN level changes over time, particularly after treatment, and may yield additional prognostic information in curative-intent radiotherapy of NSCLC. Collectively, these results have built OPN levels as an indicator for NSCLC patients.

The association between OPN overexpression and clinical outcome of NSCLC is unclear. Thus, a meta-analysis was performed to examine the effect estimate of OPN on clinical outcome. Broad search terms and multiple bibliographic databases were used in the searches to capture as many relevant articles as possible, and a robust systematic 
approach was used to select the final articles. Although we have assessed the value of OPN in NSCLC comprehensively, some limitations of our meta-analysis should not be ignored, such as patient selection, different outcome measures, and statistical techniques. ${ }^{35}$ First, potential language and risk bias were concerned, as positive research results were published more than negative ones frequently. We have tried to identify all correlative information, but several missing data are still unavoidable. Second, data on clinical outcomes were quite limited, so more articles designed to evaluate the clinical outcomes were specifically needed, and for the association of OPN with some clinical readouts, there exist differences that require further research for clarification.

\section{Conclusion}

Our meta-analysis suggested that OPN overexpression was correlated with poor survival in NSCLC, and it might provide important information for the prediction of NSCLC invasion, metastasis, and prognosis.

\section{Acknowledgments}

This work was supported by grants from the National Natural Science Foundation of China (grant number 8140010845) and Anhui Provincial Natural Science Foundation (grant number 1208085QH165).

\section{Disclosure}

The authors report no conflicts of interest in this work.

\section{References}

1. Mountain CF. Revisions in the international system for staging lung cancer. Chest. 1997;111(6):1710-1717.

2. Ishikawa M, Miyahara R, Sonobe M, et al. Higher expression of EphA2 and ephrin-A1 is related to favorable clinicopathological features in pathological stage I non-small cell lung carcinoma. Lung Cancer. 2012; 76(3):431-438.

3. Denhardt DT, Noda M, O’Regan AW, Pavlin D, Berman JS. Osteopontin as a means to cope with environmental insults: regulation of inflammation, tissue remodeling, and cell survival. J Clin Invest. 2001; 107(9):1055-1061.

4. Chuang CY, Chang H, Lin P, et al. Up-regulation of osteopontin expression by aryl hydrocarbon receptor via both ligand-dependent and ligandindependent pathways in lung cancer. Gene. 2012;492(1):262-269.

5. Zhao B, Sun T, Meng F, et al. Osteopontin as a potential biomarker of proliferation and invasiveness for lung cancer. J Cancer Res Clin Oncol. 2011;137(7):1061-1070.

6. Boldrini L, Donati V, Dell'Omodarme M, et al. Prognostic significance of osteopontin expression in early-stage non-small-cell lung cancer. $\mathrm{Br} J$ Cancer. 2005;93(4):453-457.

7. Donati V, Boldrini L, Dell'Omodarme M, et al. Osteopontin expression and prognostic significance in non-small cell lung cancer. Clin Cancer Res. 2005;11(18):6459-6465.

8. Weber GF, Lett GS, Haubein NC. Osteopontin is a marker for cancer aggressiveness and patient survival. Br J Cancer. 2010;103(6): 861-869.
9. Hu Z, Lin D, Yuan J, et al. Overexpression of osteopontin is associated with more aggressive phenotypes in human non-small cell lung cancer. Clin Cancer Res. 2005;11(13):4646-4652.

10. Schneider S, Yochim J, Brabender J, et al. Osteopontin but not osteonectin messenger RNA expression is a prognostic marker in curatively resected non-small cell lung cancer. Clin Cancer Res. 2004;10(5): 1588-1596.

11. Chang YS, Kim HJ, Chang J, Ahn CM, Kim SK, Kim SK. Elevated circulating level of osteopontin is associated with advanced disease state of non-small cell lung cancer. Lung Cancer. 2007;57(3):373-380.

12. Isa $\mathrm{S}$, Kawaguchi $\mathrm{T}$, Teramukai $\mathrm{S}$, et al. Serum osteopontin levels are highly prognostic for survival in advanced non-small cell lung cancer: results from JMTO LC 0004. J Thorac Oncol. 2009;4(9): 1104-1110.

13. Takenaka M, Hanagiri T, Shinohara S, et al. Serum level of osteopontin as a prognostic factor in patients who underwent surgical resection for non-small-cell lung cancer. Clin Lung Cancer. 2013;14(3): 288-294.

14. Wells GA, Shea B, O'Connell D, et al. The Newcastle-Ottawa Scale (NOS) for assessing the quality of nonrandomised studies in metaanalyses. Ottawa $(\mathrm{ON})$ : Ottawa Health Research Institute. Available from: www.ohri.ca/programs/clinical_epidemiology/oxford.htm. Accessed November 10, 2015.

15. Thompson SG, Higgins JP. How should meta-regression analyses be undertaken and interpreted? Stat Med. 2002;21(11):1559-1573.

16. Song F, Gilbody S. Bias in meta-analysis detected by a simple, graphical test. Increase in studies of publication bias coincided with increasing use of meta-analysis. BMJ. 1998;316(7129):471.

17. Sun BS, Li Y, Zhang ZF, You J, Wang CL. Osteopontin combined with CD44v6, a novel prognostic biomarker in non-small cell lung cancer undergoing curative resection. Ann Thorac Surg. 2013;96(6): 1943-1951.

18. Chambers AF, Wilson SM, Kerkvliet N, O'Malley FP, Harris JF, Casson AG. Osteopontin expression in lung cancer. Lung Cancer. 1996; 15(3):311-323.

19. Rud AK, Boye K, Oijordsbakken M, et al. Osteopontin is a prognostic biomarker in non-small cell lung cancer. BMC Cancer. 2013;13:540.

20. Zhang L, Hou X, Lu S, et al. Predictive significance of bone sialoprotein and osteopontin for bone metastases in resected Chinese non-small-cell lung cancer patients: a large cohort retrospective study. Lung Cancer. 2010;67(1):114-119.

21. Mack PC, Redman MW, Chansky K, et al; SWOG. Lower osteopontin plasma levels are associated with superior outcomes in advanced nonsmall-cell lung cancer patients receiving platinum-based chemotherapy: SWOG Study S0003. J Clin Oncol. 2008;26(29):4771-4776.

22. Zhang T, Zhang DM, Zhao D, Hou XM, Yang TN. Osteopontin expression is associated with platinum-based chemotherapy response and prognosis of patients with advanced non small cell lung cancer. J BUON. 2014;19(3):742-748.

23. Han SS, Lee SJ, Kim WJ, et al. Plasma osteopontin is a useful diagnostic biomarker for advanced non-small cell lung cancer. Tuberc Respir Dis (Seoul). 2013;75(3):104-110.

24. Ostheimer C, Bache M, Guttler A, Kotzsch M, Vordermark D. A pilot study on potential plasma hypoxia markers in the radiotherapy of non-small cell lung cancer. Osteopontin, carbonic anhydrase IX and vascular endothelial growth factor. Strahlenther Onkol. 2014;190(3): 276-282.

25. Yan $\mathrm{CH}, \mathrm{Lv} \mathrm{M}, \mathrm{Li} \mathrm{H}$, et al. Osteopontin is a novel prognostic biomarker in early-stage non-small cell lung cancer after surgical resection. J Cancer Res Clin Oncol. 2015;141(8):1371-1378.

26. E Y, He N, Wang Y, Fan H. Percutaneous transluminal angioplasty (PTA) alone versus PTA with balloon-expandable stent placement for short-segment femoropopliteal artery disease: a metaanalysis of randomized trials. J Vasc Interv Radiol. 2008;19(4):499-503.

27. Kim JH, Skates SJ, Uede T, et al. Osteopontin as a potential diagnostic biomarker for ovarian cancer. JAMA. 2002;287(13):1671-1679.

28. Ue T, Yokozaki H, Kitadai Y, et al. Co-expression of osteopontin and CD44v9 in gastric cancer. Int J Cancer. 1998;79(2):127-132. 
29. Furger KA, Menon RK, Tuck AB, Bramwell VH, Chambers AF. The functional and clinical roles of osteopontin in cancer and metastasis. Curr Mol Med. 2001;1(5):621-632.

30. Yu TT, Han ZG, Shan L, et al. Expression of osteopontin in non-small cell lung cancer and correlative relation with microvascular density. Asian Pac J Cancer Prev. 2014;15(1):29-32.

31. Le QT, Chen E, Salim A, et al. An evaluation of tumor oxygenation and gene expression in patients with early stage non-small cell lung cancers. Clin Cancer Res. 2006;12(5):1507-1514.

32. Fedarko NS, Jain A, Karadag A, Van Eman MR, Fisher LW. Elevated serum bone sialoprotein and osteopontin in colon, breast, prostate, and lung cancer. Clin Cancer Res. 2001;7(12):4060-4066.
33. Karapanagiotou EM, Terpos E, Dilana KD, et al. Serum bone turnover markers may be involved in the metastatic potential of lung cancer patients. Med Oncol. 2010;27(2):332-338.

34. Ostheimer C, Bache M, Güttler A, Reese T, Vordermark D. Prognostic information of serial plasma osteopontin measurement in radiotherapy of non-small-cell lung cancer. BMC Cancer. 2014;14:858.

35. Trikalinos TA, Salanti G, Zintzaras E, Ioannidis JP. Meta-analysis methods. Adv Genet. 2008;60:311-334.

\section{Publish your work in this journal}

OncoTargets and Therapy is an international, peer-reviewed, open access journal focusing on the pathological basis of all cancers, potential targets for therapy and treatment protocols employed to improve the management of cancer patients. The journal also focuses on the impact of management programs and new therapeutic agents and protocols on

\section{Dovepress}

patient perspectives such as quality of life, adherence and satisfaction. The manuscript management system is completely online and includes a very quick and fair peer-review system, which is all easy to use. Visit http://www.dovepress.com/testimonials.php to read real quotes from published authors.

\footnotetext{
Submit your manuscript here: http://www.dovepress.com/oncotargets-and-therapy-journal
} 